\title{
SON-SIRE REGRESSION BASED HERITABILITY ESTIMATES OF CHIASMA FREQUENCY, USING T7OH MOUSE TRANSLOCA- TION HETEROZYGOTES, AND THE RELATION BETWEEN UNIVALENCE, CHIASMA FREQUENCY AND SPERM PRODUCTION
}

\author{
P. de BOER and F. A. van der HOEVEN \\ Deportment of Genetics, Agricultural University, 53 Gen. Foulkesweg, \\ Wageningen, The Netherlands
}

Received 22.ii.77

\begin{abstract}
SUMmary
$\mathrm{T}(1 ; 13) 70 \mathrm{H} /+$ translocation heretozygous mice were used for assessing heritability values for chiasma frequencies and the epididymal sperm count. The chiasma frequency estimates were based on 15 son-sire pairs, the translocation heterozygotes being maintained in a Swiss random-bred genetic background. The chiasma frequencies were scored separately for the $\mathrm{T} 70 \mathrm{H} /+$ derived multivalent, specific pairing segments within the multivalent and the remaining bivalents. Chiasma counts within these specified parts of the genome were positively correlated. The heritability estimates, significantly greater than zero, ranged from $0.78-0.98$, depending on the chromosome segments included. These results indicate a strong genetic control on a cellular basis for the formation of chiasmata in the mouse. Despite significantly positive correlations and regressions between the various chiasma frequencies and the sperm count (for which 29 pairs of observations were available), no significant heritability estimate for the sperm count was obtained. The relation between the chiasma frequency and the sperm count was weakest when the chiasma count was confined to a region of the translocation-caused multivalent in which the absence of a chiasma almost always resulted in the production of an univalent. This indicates that in the translocation heterozygotes used, the overall chiasma frequency has a greater predictive value for the sperm count than autosomal univalence alone.
\end{abstract}

\section{INTRODUCTION}

IT has been generally stated that the genetic process of crossing-over, which leads to chiasmata observed during diplotene, diakinesis and metaphase of the first meiotic division, is influenced by genes (for review, see Bodmer and Parsons, 1962; Sybenga, 1975). When the production of cross-overs is regarded as a two-step event, one step being the pairing of homologues (pre-conditioning for exchange), and the other step, the actual occurrence of a chiasma, it is thought that genes influence both (for a discussion, see Jones, 1974). Genes of crucial importance for the process are inferred through meiotic mutants. Another approach demonstrating the influence of genes is using the ratio of the additive genetic variance to the total phenotypic variance (the heritability or $h^{2}$ ). For this purpose, one can either compare individuals of different consanguinity, or select for low and/or high chiasma frequency (Chinnici, 1971; Shaw, 1972). The latter approach will produce an assessment of the " realised- $h^{2}$ " (Shaw, 1972). The genetic 
nature can also be assessed by producing an F2 generation of selfing plants, the parental types differing in chiasma frequency and their phenotypes not being recovered among the F2, or by analysing different inbred lines for the character (Rees, 1955).

$\mathrm{Up}$ to now, the contribution of the genes to the manifestation of chiasmata has been demonstrated in fungi, insects, plants, mammals and is suggested for man (Hulten et al., 1970).

We have attempted to make an assessment of the $h^{2}$ value, using a sonsire regression in a stock of translocation heterozygous mice $(\mathrm{T}(1 ; 13) 70 \mathrm{H} /+)$ using both chiasma counts within the translocation-caused multivalents and counts in the bivalents.

Autosomal univalents, usually rarely observed in mammalian meiosis, can be generated through translocation heterozygosity (Lyon and Meredith, 1966). A relation between univalence and a breakdown of meiosis has been suggested both for the mouse and for man (Miklos, 1974; Searle, 1974; Chandley et al., 1975). We have investigated this point further by comparing the epididymal sperm count with the counts for translocation-caused univalence and the chiasma counts, using the son-sire pairs of this experiment.

\section{Materials and methods}

The mice used were male $\mathrm{T}(1 ; 13) 70 \mathrm{H}$ translocation heterozygotes $(\mathrm{T} /+)$, which had been outcrossed for an average of 10 generations to a Swiss random-bred stock $(\mathrm{Cpb}(\mathrm{SE}) \mathrm{S}$ ) of sufficient size to avoid inbreeding. A total of 15 unrelated families has been obtained by this procedure and from each family, a $\mathrm{T} /+$ father and a $\mathrm{T} /+$ son entered the experiment. Meiotic preparations were made according to an air-drying technique (Evans et al., 1964), and the chromosomes were $C$-banded by the BSG technique of Sumner (1972). One hundred diplotene-diakinesis cells per male were scored from one testis (right or left) and the stage of meiosis assessed as described previously (de Boer and Groen, 1974; de Boer, 1976). Cells of class 3 , containing the most contracted bivalents were not included. In all 100 cells, the translocation-caused multivalent configuration was scored and notations of RIV, GIV, CIII + I follow those explained by de Boer (1976), who gives photomicrographs as well. Fig. 1 gives diagrams of configurations most frequently observed with the notation for whole chromosomes and their interstitial $(i)$ and translocated $(t)$ segments. It also gives their overall frequencies. A few cells (5) were found where chiasmata were lacking in both interstitial segments of the pachytene cross, thus giving rise to two heteromorphic bivalents. For 10 cells out of the sample of 100 , the chiasma frequency of all the autosomal bivalents made up by homologous chromosomes (17 in total) was scored. Both observers contributed equally to each male scored.

At the time of autopsy, spermatozoa from the capita epididymes were counted according to a method described by Searle and Beechey (1974). The males were 6-8 months old when killed, and had been in the continuous presence of a female for 3-5 months, which resulted in the production of 3-4 litters. Gare has been taken that young, if present, were at least 5 days old at the time of autopsy of their father, in order to avoid any influence of the post-parturition copulation on the sperm count. 


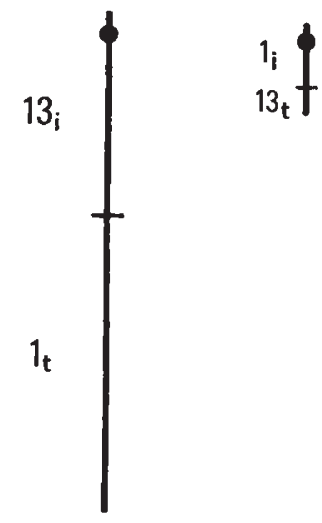

$13^{1}$
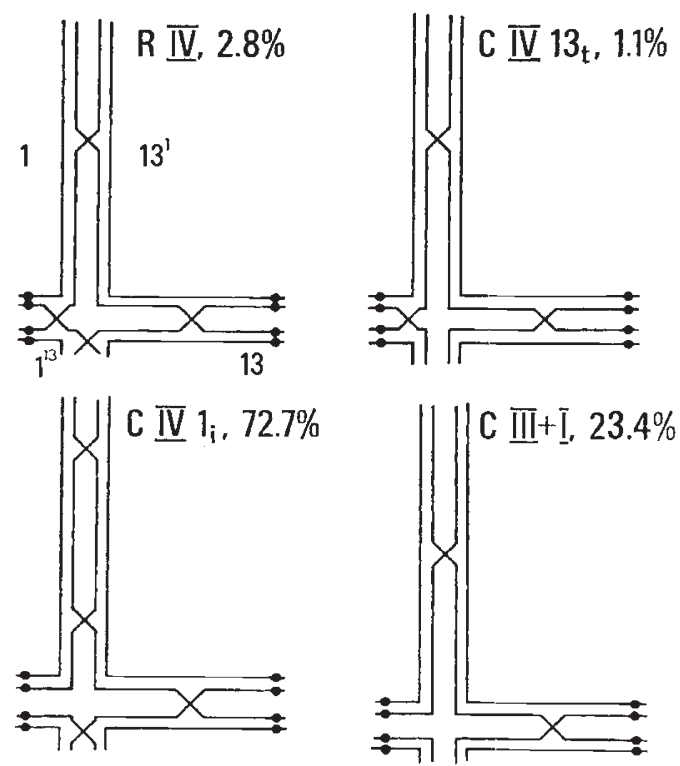

FIG. 1.-Diagrams of the multivalent configurations encountered in the $T(1 ; 13) 70 \mathrm{H}$ reciprocal mouse translocation together with the notations of the chromosomes involved and their interstitial and translocated seginents. Segment $1_{t}$ may have 1 or 2 chiasmata. The overall frequency of each configuration is also given $(n=2995)$. For an explanation of the symbols RIV, CIV13t etc., see de Boer (1976).

\section{RESULTS}

Within the $\mathrm{T} /+$ caused multivalent included in the analysis, either 1 or 2 chiasmata were present in segment $l_{t}, 0$ or 1 in segments $13_{i}$ and $l_{i}$ and 1 in segment $13_{i}$. The following symbols referring to chiasma counts per 100 cells will be used:

Chl: The number of multivalents with 2 chiasmata in $1_{t}$;

Ch2: The number of multivalents with 0 chiasmata in $13_{t}$ and 0 in $1_{i}$, thus producing a univalent for chromosome $1^{13}$. Ch2 equals the frequency of translocation-caused univalents and almost equals the frequency of cells with no chiasma in $13_{t}$ (adding CIV13 fig. 1);

Ch3: The total number of chiasmata in all 100 multivalents;

Ch4: The total number of chiasmata in normal bivalents, summed over the 10 cells scored in this respect; and

Ch5: The sum of Ch3 and Ch4.

Table 1 gives the means and standard deviations for each parameter thus calculated and for the sperm count (Sp C). The sperm count data are based on the sum of the counts of both epididymes. Within animals, there is a good correlation between the counts of both epididymes with an intraclass correlation coefficient $r=0.79(n=43)$. Of the total number of cells scored 18.3 per cent were considered to be late diplotene or early diakinesis (Class 1) and $81 \cdot 7$ per cent were of class 2 (mid-diakinesis). 
TABLE 1

Means ( $\overline{\mathbf{x}})$ and standard deviations (S.D.) of the chiasma parameters (Ch1-5) and sperm count (Sp C) scored. The data are pooled for fathers and sons. For an explanation of the chiasma parameters, see the Results. The mean chiasma frequencies per cell for the chromosome segments or chromosomes indicated are given as well $(\bar{y})$

\begin{tabular}{lrrr} 
& \multicolumn{1}{c}{$\bar{x}$} & \multicolumn{1}{c}{ S.D. } & \multicolumn{1}{c}{$\bar{y}$} \\
Ch1 & $28 \cdot 63$ & $9 \cdot 57$ & $1 \cdot 29$ \\
Ch2 & $24 \cdot 53$ & $13 \cdot 69$ & $0 \cdot 77$ \\
Ch3 & $306 \cdot 53$ & $20 \cdot 75$ & 3.07 \\
Ch4 & $210 \cdot 47$ & $12 \cdot 22$ & $21 \cdot 05$ \\
Ch5 & $517 \cdot 00$ & $31 \cdot 12$ & $24 \cdot 12$ \\
Sp C & $420 \cdot 20$ & $121 \cdot 84$ &
\end{tabular}

The effect of meiotic stage on chiasma number as scored in segments 1 , and $13_{t}$ is depicted in table 2. As found earlicr (Forejt, 1972; de Boer, 1976) the numbers drop when meiosis proceeds. The males used were homogeneous, however, with respect to the numbers of class 1 and class 2 primary spermatocytes, thereby not biasing a comparison between fathers and sons $\left(\chi^{2}{ }_{29}=36.56\right.$ n.s. $)$.

A correlation matrix of the parameters used (Chl-5 and the sperm count) is given in table 3 .

TABLE 2

The effect of the stage of meiosis (class 1 containing late diplotene and early diakinesis and class 2 containing mid diakinesis) on the number of chiasmata scored in multivalents

No.of chiasmata

Segment $1_{t}$

1

2

Segments $1_{i}$ plus $13_{t}$ At least 1 0

\begin{tabular}{|c|c|c|c|c|}
\hline \multicolumn{4}{|c|}{ Spiralisation class } & \multirow[b]{3}{*}{$\begin{array}{l}\chi_{1}{ }^{2}=27.85 \\
\mathbf{P}<0.005\end{array}$} \\
\hline \multicolumn{2}{|c|}{1} & \multicolumn{2}{|c|}{2} & \\
\hline $\begin{array}{l}341 \\
208\end{array}$ & $\begin{array}{l}62 \cdot 1 \% \\
37 \cdot 9 \%\end{array}$ & $\begin{array}{r}1795 \\
651\end{array}$ & $\begin{array}{l}73.4 \% \\
26.6 \%\end{array}$ & \\
\hline 476 & $86 \cdot 7 \%$ & 1818 & $74.3 \%$ & $x_{1}^{2}=38.32$ \\
\hline 73 & $13.3 \%$ & 628 & $25 \cdot 7 \%$ & 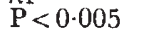 \\
\hline
\end{tabular}

TABLE 3

Correlations between the various chiasma parameters used and between the chiasma parameters and the sperm count, each correlation involving 29 or 30 pairs. $P$ gives the probability of the correlation being greater than zero (for the negative ones) and smaller than zero (for the positive ones). The symbols follow those explained in the Results

$\begin{array}{lccc} & \text { Ch2 } & \text { Ch4 } & \text { Sp C } \\ \text { Ch1 } & -0.62 & 0.74 & 0.63 \\ & \mathbf{P}=0.0004 & \mathbf{P}=0.0001 & \mathbf{P}=0.0004 \\ \text { Ch2 } & & -0.51 & -0.36 \\ & & \mathbf{P}=0.0041 & \mathbf{P}=0.0468 \\ \text { Ch3 } & 0.67 & 0.53 \\ & & \mathbf{P}=0.0002 & \mathbf{P}=0.0028 \\ \text { Ch4 } & & 0.49 \\ \text { Ch5 } & & \mathbf{P}=0.0066 \\ & & & 0.56 \\ & & & \mathbf{P}=0.0016\end{array}$


Correlations which were partially or wholly autocorrelations have been left out. High chiasma frequencies in segment $1_{t}$ go accompanied with high chiasma frequencies in segment $13{ }_{t}$. Animals, which had a high chiasma score in the multivalents also had a high score in the bivalents, suggesting cellular control of the phenomenon. Correlations between all chiasma parameters and the epididymal sperm count were such that high sperm counts were more likely to occur in animals with a high chiasma count.

When the chiasma data of the 15 sons were regressed on to their 15 fathers, the following picture emerged (table 4): regression coefficients were significantly larger than zero for Chl, 3, 4 and 5. For the sperm count

TABLE 4

Regression coefficients and heritability estimates $\left(\mathrm{h}^{2}=2 \mathrm{~b}\right.$ for a son-sire regression) for all chiasma parameters used and for the sperm count

$\begin{array}{lcccc} & n & b & \mathrm{P}, b>0 & h^{2} \\ \text { Ch1 } & 15 & 0 \cdot 49 \pm 0 \cdot 25 & 0 \cdot 025<\mathrm{P}<0 \cdot 05 & 0 \cdot 98 \pm 0 \cdot 50 \\ \text { Ch2 } & 15 & 0 \cdot 28 \pm 0 \cdot 16 & \text { n.s. } & 0 \cdot 56 \pm 0 \cdot 32 \\ \text { Ch3 } & 15 & 0 \cdot 39 \pm 0 \cdot 20 & 0 \cdot 025<\mathrm{P}<0 \cdot 05 & 0 \cdot 78 \pm 0 \cdot 40 \\ \text { Ch4 } & 15 & 0 \cdot 44 \pm 0 \cdot 13 & \mathrm{P}<0 \cdot 005 & 0 \cdot 88 \pm 0 \cdot 26 \\ \text { Ch5 } & 15 & 0 \cdot 48 \pm 0 \cdot 17 & 0.005<\mathrm{P}<0 \cdot 01 & 0 \cdot 96 \pm 0 \cdot 34 \\ \text { Sp C } & 34 & 0 \cdot 29 \pm 0 \cdot 18 & \text { n.s. } & 0 \cdot 58 \pm 0 \cdot 36\end{array}$

data, a larger number of father-son pairs were available and the regression coefficient was not significantly larger than zero. The ensuing heritability estimates, $\frac{\text { additive genetic variance }}{\text { phenotypic variance }}=\frac{V A}{V P}$ are rather high, though with large standard deviations.

The material was also used for assessing the relation between the chiasma data, of which Ch2 represents the fraction of translocation-caused univalence, and the sperm count data, remembering the significantly positive or negative correlations between the two (table 3 ). For each chiasma parameter tested (the sperm count being dependent variable), a linear first power equation $(Y=a+b X)$ gave the best fit to the data when tested against higher power equations $\left(Y=a+b X+c X^{2}\right.$ etc). The regression lines are given in table 5 and two depicted in fig. 2. All values of $b$ are significantly greater or smaller than zero.

\section{TABLE 5}

The best lines of fit (using additive models) of the relation between the chiasma count (X) and the sperm count (the dependent variable, Y)

$\begin{array}{llll}\text { For Ch2 } & r=494.92-3.20 X & s_{b}=1.56 & \mathrm{P} \sim 0.025 \\ \text { For Ch3 } & r=-498 \cdot 00+3.00 X & s_{b}=0.90 & P<0.005 \\ \text { For Ch4 } & r=-598 \cdot 16+4.84 X & s_{b}=1.65 & 0 \cdot 005<\mathrm{P}<0.01 \\ \text { For Ch5 } & Y=-712.35+2 \cdot 19 X & s_{b}=0.60 & \mathrm{P}<0.005\end{array}$

\section{Discussion}

It should be remembered that the main parameter used in this investigation, i.e. chiasma count, is not fixed during the stages of meiosis, used for scoring, but drops as metaphase approaches. So the $h^{2}$ values derived 

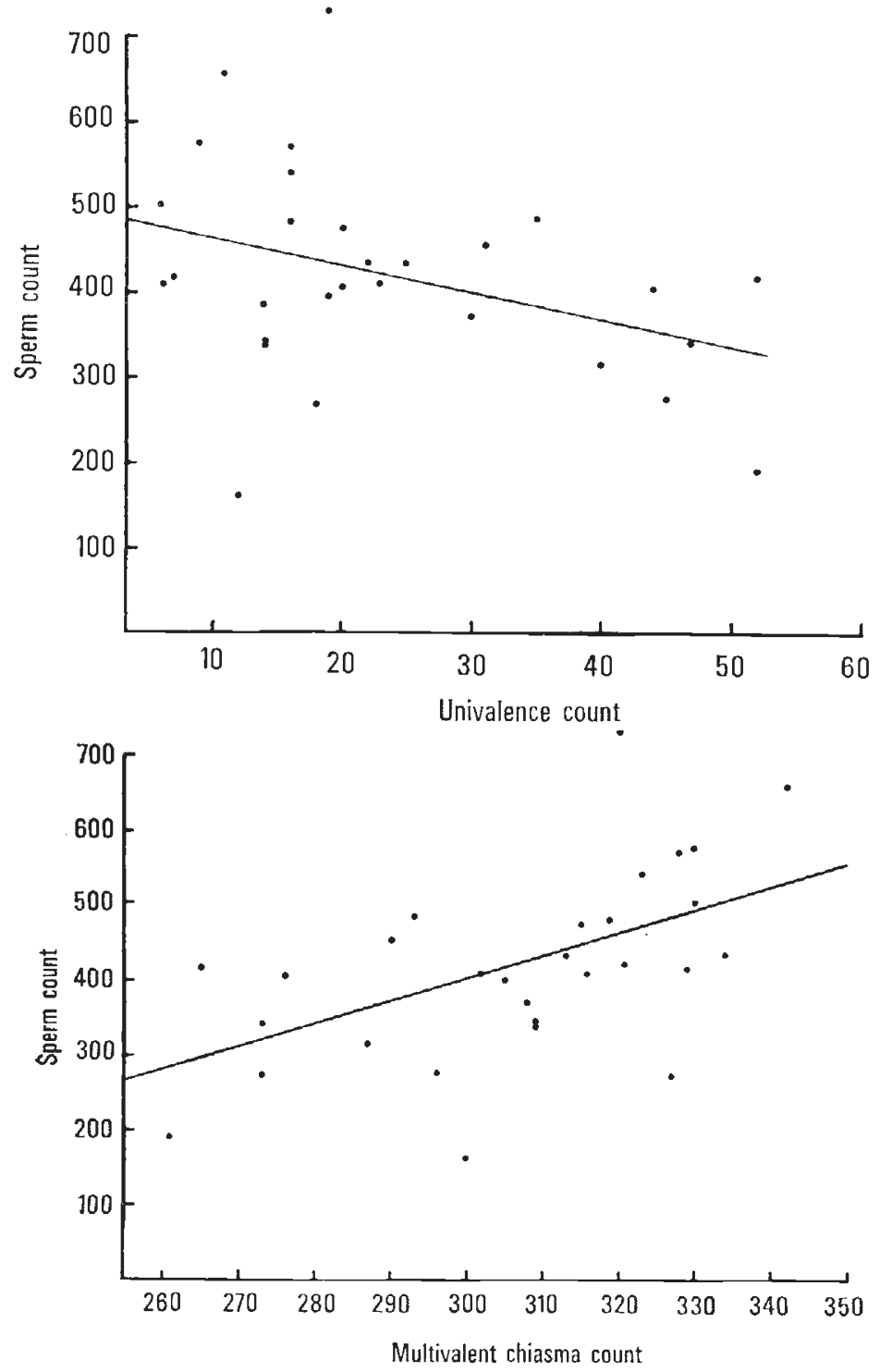

Frc. 2.---.The lincs of best fit for the relation between the epididymal sperm count and translocation-caused univalence (upper graph) and for the sperm count and the total multivalent chiasma counts (lower graph).

contain a component of chiasma terminalisation. If the number of terminalised chiasmata is linearly related to the total number of chiasmata originally present, then the values given are the correct ones for chiasma formation. It is more likely that counts made in animals with a high intrinsic chiasma value, are more biased by the process of chiasma tcrminalisation because of increasing tension within the bivalent, when spiralisation proceeds. Even then, the slope of the regression line should not be altered. 
We have tried to limit the terminalisation-component by omitting late diakinesis and metaphase I from the scores.

The $h^{2}$ values obtained are very high and apply both to the multivalent and to the autosomal bivalents, the two of which were positively correlated. Despite the large deviations, the conclusion is warranted that under normal animal house conditions there is still considerable phenotypic variance with respect to chiasma frequency, which is mainly due to additive genetic factors. Thus, the contribution of intra-locus (or dominance) variance cannot be very high, which is in line with results by Shaw (1974) in Schistocerca gregaria. A genetic contribution to chiasma frequency in the mouse could already be inferred from differences in chiasma counts between different inbred strains of mice (Kyslikova and Forejt, 1972). Also, the result of Berry et al. (1973) that the hybrid males of a cross between Skokholm wild mice and laboratory mice exhibited chiasma counts intermediate to the parental ones, can be taken to indicate genetic control. Our results also show that because of the high $h^{2}$-values selection for high and low chiasma frequency is feasible using the Swiss random-bred stock. Shaw (1972) using the locust Schistocerca gregaria showed values for the realised $h^{2}$ of 0.27 for upward selection and 0.48 for downward selection, values either suggesting a less stable environment in their experiments or a less strict control of chiasma formation in this animal.

Univalence arising from a very low chiasma count could eventually influence the fitness of the individual, but above a minimum of one chiasma per bivalent, an optimum count (with regard to fitness) seems not very sharply defined and under animal house circumstances, selection, narrowing the genetic variance, seems hardly important if present at all.

Findings of Lyon and Meredith (1966) and Searle (1974) suggest that mouse translocations characterised by a high likelihood of producing autosomal univalents are prone to cause a breakdown of spermatogenesis mostly before the secondary spermatocyte stage. According to this criterion, the $\mathrm{T} 70 \mathrm{H} /+$ male translocation carrier should be sterile, but it is not. We could, however, establish a significant correlation and regression between univalance (translocation induced) and epididymal sperm count. Correlations and regressions became stronger when chiasma counts were included covering a greater part of the genome. It is impossible to tell whether the relationship between the two is causative or whether both are influenced by the same genes. If one likes to apply Miklos' (1974) hypothesis regarding spermiogenic arrest one could state that a high chiasma frequency results from enough meiotic pairing sites being occupied. Unoccupied sites, according to the hypothesis, would lead to decondensation of chromatin sometime afterwards. Decondensation followed by unwanted transcription (at least in Drosophila) then endangers the lives of the spermatids concerned, thereby lowering the sperm count. In the mouse, however, there is intense RNA synthesis during middle pachytene (Monesi, 1965), when chiasma formation is complete.

Cohen (1973) maintains that there is a positive correlation between sperm redundancy (the number of sperm ejaculated to fertilise a single egg) and chiasma count, covering a great diversity of species with totally different reproductive physiology. Within related species, Wallace (1974) could not find such a relation.

In the mouse, epididymal sperm counts reflect the size of the ejaculate 
and hence the number of spermatozoa in the oviduct at the time of fertilisation (de Boer et al., 1976). We found a relation between sperm count and chiasma count and it is difficult to attach a biological significance to it, especially when univalents are not involved (see tables 3 and 5). The negative relation found for autosomal univalents and the sperm count could be explained on the basis of univalents interfering with proper anaphase I segregation. It has already been demonstrated (de Boer, 1976) that $\mathrm{T} 70 \mathrm{H} /+$ has a slightly decreased sperm count and also that the T70H-derived univalent was in the majority of cases behaving regularly (not causing numerical non-disjunction) during anaphase $\mathrm{I}$. When the correlations given in table 3 between the various chiasma frequencies and the sperm count are examined again, the absolute value of the correlation is lowest when the formation of a univalent is measured (Ch2). In fact the correlation of Chl (giving the number of chiasmata in segment $l_{t}$ ) with the sperm count is significantly greater than the Ch2-Sp C correlation (using transformation of $r$ to $z, \mathrm{P}<0.05$ ). This suggests that increased univalence is less important for lowering the sperm count than an overall decrease in the chiasma frequency throughout the whole of the genome, thereby reinforcing the observations cited before that the T70H-derived univalent is able to coorientate with the other chromosomes of the translocation complex. Thus, data presented here do not prove that a single univalent can cause spermatocyte death between metaphase I and metaphase II. The possibility is left open that chiasma count, univalence, spermatocyte break-down and sperm count are influenced by common genes.

Acknowledgments. - Thanks to Mr James Davies and Mr Norman Davidson of the Medical Research Council, Clinical and Population Cytogenetics Unit, Edinburgh, for help with the computations and preparation of the figures.

\section{REFERENCES}

BERRY, R. J., BEEChEY, C. V., AND SEARLE, A. G. 1973. Cytogenetic radiosensitivity and chiasma frequency in wild and laboratory mice. Mutation Res., 19, 129-131.

BODMER, W. F., AND Parsons, P. A. 1962. Linkage and recombination in evolution. Adv. Genet., 11, 1-100.

BOER, P. DE. 1976. Male meiotic behaviour and male and female litter size in mice with the $\mathrm{T}(2 ; 8) 26 \mathrm{H}$ and $\mathrm{T}(1 ; 13) 70 \mathrm{H}$ reciprocal translocations. Genet. Res., 27, 369-387.

BOER, P. DE, AND GROEN, A. 1974. Fertility and meiotic behaviour of male T70H tertiary trisomics of the mouse (Mus musculus). A case of preferential telomeric meiotic pairing in a mammal. Cytogenet. Cell Genet., 13, 489-510.

BOER, P. DE, hOEVEN, F. A. VAN DER, AND CHARDON, J. A. P. 1976. The production, morphology, karyotypes and transport of spermatozoa from tertiary trisomic mice and the consequences for egg fertilization. 7. Reprod. Fert., 48, 249-256.

CHANdLey, A. C., EDMOND, P., CHRISTIE, s., GOWANS, L., Fletcher, J., FRACKIEWICZ, A., AND NEWTON, M. 1975. Cytogenetics and infertility in man. I. Karyotype and seminal analysis. Ann. Hum. Genet., Lond., 39, 231-254.

ChinnicI, J. P. 1971. Modification of recombination frequency in Drosophila. 1. Selection for increased and decreased crossing over. Genetics, 69, 71-83.

CoHen, J. 1973. Cross-overs, sperm redundancy and their close association. Heredity, 31, $408-412$.

EVANS, E. P., BRECKON, C., AND FORD, C. E. 1964. An air-drying method for meiotic preparations from mammalian testes. Cytogenetics, 3, 289-294.

Forejt, J. 1972. Chiasmata and crossing-over in the male mouse (Mus musculus). Suppression of recombination and chiasma frequencies in the ninth linkage group. Folia Biol., 18, 161-170. 
HUlten, M., EliAsSON, R., AND TILlinger, K. G. 1970. Low chiasma count and other meiotic irregularities in two infertile $46, \mathrm{XY}$ men with spermatogenic arrest. Hereditas, 65 , 285-290.

JONES, G. H. 1974. Correlated components of chiasma variation and the control of chiasma distribution in rye. Heredity, 32, 375-387.

KYSLIKOVA, L., AND FOREJT, J. 1972. Chiasma frequency in three inbred strains of mice. Folia Biol., 18, 216-218.

LYON, M. F., AND MEREDITH, R. 1966. Autosomal translocations causing male sterility and viable aneuploidy in the mouse. Cytogenetics, 5, 335-354.

miklos, G. L. G. 1974. Sex-chromosome pairing and male fertility. Cytogenet. Cell Genet., $13,558-577$.

MONEsI, v. 1965. Synthetic activities during spermatogenesis in the mouse. Exp. Cell Res., $39,197-224$.

REES, H. 1955. Genotypic control of chromosome behaviour in rye. I. Inbred lines. Heredity, 9, 93-116.

SEARLE, A. G. 1974. Nature and consequences of induced chromosome damage in mammals. Genetics, 78, 173-186.

SEARLE, A. G., AND BEECHEY, c. v. 1974. Sperm-count, egg-fertilization and dominant lethality after X-irradiation of mice. Mutation Res., 22, 63-72.

SHAw, D. D. 1972. Genetic and environmental components of chiasma control. II. The response to selection in Schistocerca. Chromosoma, 37, 297-308.

SHAW, D. D. 1974. Genetic and environmental components of chiasma control. III. Genetic analysis of chiasma frequency variation in two selected lines of Schistocerca gregaria Forsk. Chromosoma, 46, 365-374.

SUMNER, A. T. 1972. A simple technique for demonstrating centromeric heterochromatin. Exp. Cell Res., 75, 304-306.

sybenGA, J. 1975. Meiotic configurations. Springer-Verlag, Berlin, Heidelberg, New York.

wallace, H. 1974. Chiasmata have no effect on fertility. Heredity, 33, 423-428. 